\title{
Effects of Scald Injuries on Liver and Brain Adult Male Albino Rats (Biochemical, Histopathological and Immunohistochemical Study)
}

\author{
Heba El Sayed Mostafa, Eman Ahmed Alaa El-Din', and Maha Zayed Mohammed \\ Hassan²
}

${ }^{1}$ Department of Forensic Medicine and Clinical Toxicology
${ }^{2}$ Department of Histology and Cell Biology

Faculty of Medicine, Zagazig University, Zagazig, Egypt

\begin{abstract}
Objectives: Thermal injuries still occur frequently in many parts of the world. Severe cutaneous thermal injuries are associated with multiple organ dysfunctions, which demand urgent medical interventions to ensure patient's survival. This failure of different organ systems can be attributed to uncontrolled production of inflammatory cytokines such as interleukin-6 (IL-6), tumor necrosis factor-alpha (TNF- $\alpha$ ), interleukin 1beta (IL-1 $\beta$ ). The purpose of this study was to detect the effects of scald injuries on liver and brain of adult male albino rats at 6,12 and 24 hours post injury. Material and methods: Fifty four adult male albino rats were equally divided into three groups: group I (negative control group), group II (sham group) and group III (scald injured group) effects of scald injuries on liver and brain at 6,12 and 24 hours post injury were studied through: estimation of serum aspartate aminotransferase (AST), alanine aminotransferase (ALT) and Alkaline phosphatase (ALKP) levels and histopathological examination using light microscope including HandE stained sections and immunohistochemical staining of sections for detection of (TNF- $\alpha$ ) activity.Results: In group III (scald injured group), a markedly affected and disturbed liver functions represented by significant increases in serum AST, ALT and ALKP levels, histopathological changes were (aggregations of inflammatory cells, congestion and dilatation of blood vessels, vaculated hepatocytes with darkly stained nucleui and hydropic degeneration) and ( shrunken neurons with pyknotic nuclei, vacuolated cytoplasm and edema) .Time dependent TNF- $\alpha$ activation was detected in liver and brain at $6 \mathrm{~h}$ post injury lasting till 24h.Conclusion: From the previous results it can be concluded that, scald injury induced a marked affection and disturbance in liver functions, as characterized by significant increases in serum ALT, ASTand ALKP, histopathological changes in liver and brain. Also, a time dependent TNF- $\alpha$ activation was detected in liver and brain of adult male albino rats.
\end{abstract}

Keywords Thermal injury, multiple organ dysfunctions, liver function, TNF- $\alpha$

\section{Introduction}

$\mathrm{T}$ Thermal injuries represent a significant cause of death and disability around the world. Scald injuries are an important public health issue since most are preventable, yet cause considerable morbidity and mortality. They can be associated with significant pain and prolonged treatment with some physical and psychological effects lasting a lifetime. Scalds account for one-third to one-half of all scalds in high and middle income countries and globally account for approximately $5 \%$ of all scald-related deaths, with pre-school children experiencing a disproportionately high mortality rate compared to other age groups (Shah et al., 2013).

A scald refers to tissue damage from hot liquids, usually water. Other hot fluids include oils, molten rubber, other liquid chemicals and steam. The water scald is a common domestic accident, especially to children and old people (Agay et al., 2008).

Severity of scald is related to the depth and percentage of the affected body surface area (TBSA) of the animal. Third degree scald (TDB) is also known as a full thickness scald which destroys all the epidermal and dermal 
skin layers. The tissue damage extends below hair follicles and sweat glands to subcutaneous fat tissue. TDB is usually not painful because the injury has destroyed nerve endings (Adeteye et al., 2011).

A significant incidence of multiple organ dysfunction (MODS) and failure (e.g. liver, thymus, spleen, skeletal muscle, lung, heart, and brain) has been noted in both animals and humans, surviving the initial insult of severe thermal injury (Zhang et al., 2008).

The liver is thought to be a major organ responsible for initiating MODS. Because of its important role in metabolism, homeostasis, and host defense mechanisms. Also, it plays a central role after scalds as a target for remote organ dysfunction in systemic inflammation. It is not surprising, therefore, that patients with pre-existing liver disease have an almost doubled risk of mortality compared with scalded patients with similar histories, injuries, and medical profiles (Price et al., 2007).

Thermal injury is characterized by increased microvascular permeability, which causes massive fluid volume requirements during resuscitation. Thermal injury to peripheral tissue often causes systemic reactions, such as fever, hyperalgesia, anorexia, and increased permeability of the blood-brain barrier (BBB). Generalized encephalopathy is the most common neurologic complication of thermal injury in children occurring with a $14 \%$ incidence (Swann et al., 2007).

Cytokines are important mediators in the post scald pathophysiological process. Notably, the cytokine cascade associated with scald-induced oxidative stress. Furthermore, it has been shown that scald injury is associated with reactive oxygen species (ROS) release and lipid peroxidation, which causes oxidative damage to cellular membranes and ultimately leads to cell death (Agay et al., 2008).

The early appearance of inflammatory cytokines in the systemic circulation, including tumor necrosis factor- $\alpha$ (TNF- $\alpha$ ), interleukin-1 (IL-1), and interleukin-6 (IL-6), was demonstrated following thermal injury both in humans and animals (Wang et al., 2011).

Tumor necrosis factor alpha (TNF- $\alpha)$ is believed to be the initiating cytokine that induces a cascade of secondary cytokines and humoral factors that can lead to local and systemic sequelae. Moreover, TNF- $\alpha$ is a potent mediator of the shock-like state associated with severe scald and sepsis (Chen et al., 2011).

This study aimed to evaluate the effects of scald injuries on liver and brain of adult male albino rats at 6,12 and 24 hours post injury through studying the following parameters; estimation ofserum aspartate aminotransferase (AST), alanine aminotransferase (ALT) and Alkaline phosphatase (ALKP) levels and histopathological examination by using $\mathrm{H} \& \mathrm{E}$ stained sections and immunohistochemical staining for detection of tumor necrosis factor alpha (TNF- $\alpha)$ activity.

\section{Material and Method}

\section{A- Material}

\section{1-Animals}

Fifty four adult male albino rats, weighing $150-200 \mathrm{gm}$, were used in this study. The animals were kept in standard housing conditions and were freely supplied with food and water for one week before the experiment, for acclimatization.

\section{2-Experimental Design}

Each animal was anesthetized and given analgesic with an intraperitoneal injection of ketamine hydrochloride $(20 \mathrm{mg} /$ $\mathrm{kbw})$ and diazepam $(0.1 \mathrm{mg} / \mathrm{kbw})$. The back and flank skin of the rats were shaved. Rats were placed in supine position in a plaster cast with an area of their backs exposed through an opening in the cast, immersed in a hot water bath $\left(100^{\circ} \mathrm{c}\right)$ for 10 seconds (Jewo et al., 2011). This should produce non lethal full thickness injury to the skin that covers $20 \%$ of the total body surface area which were calculated by using the formula of Lee which is: Total body surface area $($ TBSA $)=($ body weight in grams $\mathrm{x} 0.78)+148$ (Farahat, 1999). TBSA of a rat weighing 300 grams is $382 \mathrm{~cm}^{2}$. So that $20 \%$ of TBSA equals $76.4 \mathrm{~cm}^{2}$.

\section{Grouping of animals}

The study was carried out on 54 adult male albino rats. They were divided into three equal groups, 18 rats in each group.

- Group I (negative control group): Rats received only regular diet and tap water to measure the basic parameters.

- Group II (sham group): Rats were anesthetized and given analgesic (with an intraperitoneal injection of ketamine hydrochloride $20 \mathrm{mg} / \mathrm{kbw}$ and diazepam 0.1 $\mathrm{mg} / \mathrm{kbw}$ ) and shaved, then put belly up in tap water for 10 seconds and removed.

- Group III (scald injured group):Rats were anesthetized and given analgesic (with an intraperitoneal injection of ketamine hydrochloride $20 \mathrm{mg} / \mathrm{kbw}$ and diazepam 0.1 $\mathrm{mg} / \mathrm{kbw}$ ) and shaved, then immersed into hot water bath $\left(100^{\circ} \mathrm{c}\right)$ for 10 seconds to produce full thickness injury to the skin (Jewo et al., 2011).

At the end of the specified durations (6, 12 and 24 hours), six rats for each time point from each group were anesthetized and subjected to blood sampling for estimation of AST, ALT and ALKP levels, then they were sacrificed, the liver and brain were subjected to histopathological 
examination using $\mathrm{H} \quad \& \quad \mathrm{E}$ stained sections and immunohistochemical staining for TNF- $\alpha$ activity.

\section{B- Methods}

\section{1- Biochemical Study}

Venous blood samples were collected from the retro-orbital plexus of the animals by capillary glass tubes according to procedure described by Nemzek et al. (2001)for measuring of serum aspartate aminotransferase (AST), alanine aminotransferase (ALT) and Alkaline phosphatase (ALKP) levels.

\section{2- Histopathological examination}

\section{Hematoxylin and Eosin stain}

The brain and liver specimens from each group were fixed immediately in a $10 \%$ neutral-buffered formalin solution and processed for light microscopic study to obtain paraffin sections of $5 \mu \mathrm{m}$ thickness. They were stained with $\mathrm{H} \& \mathrm{E}$ (Bancroftand Gamble, 2002).

\section{Immunohistochemical study}

Immuno-staining was performed using the avidinbiotin peroxidase technique for localization ofTNF- $\alpha$. Paraffin sections mounted on coated slides were deparaffinized and treated with $0.01 \mathrm{M}$ citrate buffer for 10 minutes to unmask antigens. Then sections were incubated in $\mathrm{H} 2 \mathrm{O} 2$ for 10 minutes to abolish endogenous peroxidase activity before blocking with $5 \%$ horse serum for $2 \mathrm{hrs}$ at room temperature to inhibit the nonspecific immunoreactions. Primary monoclonal anti- TNF- $\alpha$ antibody[52B83] was applied at 1:5000 dilutions. Sections were incubated with primary monoclonal antisera for $36 \mathrm{~h}$ at $4^{\circ} \mathrm{C}$. After washing they were incubated with biotinylated secondary antibodies for $5 \mathrm{hrs}$ and then followed by avidin-biotin peroxidase complex. Finally immune reaction was visualized with $0.05 \%$ diaminobenzidine. Then the slides were counter stained with Mayer's hematoxylin before mounting (Happerfield et al., 1993).

\section{3- Statistical analysis}

For statistical analysis, SPSS 13.0 for windows program was used. Data was represented as means $\pm \mathrm{SD}$. The differences were compared for statistical significance by ANOVA. Difference was considered significant at $\mathrm{p} \leq 0.05$ and Chi square $\left(\mathrm{X}^{2}\right)$ was used to test the association between 2 variables for qualitative data (number and percent).

\section{Results}

All the parameters of both control groups were within normal and there were no statistically significant differences between them all over the periods of the study.

\section{1- Biochemical results (Table-1)}

Group III (scald injured group) showed a marked affection and disturbance in liver functions represented by significant increases in serum AST, ALT and ALKP levels $(\mathrm{p} \leq 0.05)$ as compared to control groups (group I and group II). Also, serum levels of AST, ALT and ALKP in group III showed a significant increase $(\mathrm{p} \leq 0.05)$ from 6 hours post scald injury $(62.1 \pm 9.19, \quad 50.1 \pm 2.13$ and $78 \pm 8.51 \mathrm{IU} / \mathrm{L})$ up to 24 hours $(131.3 \pm 4.64,120.9 \pm 2.45$ and $105.8 \pm 7.76 \mathrm{IU} / \mathrm{L})$ respectively.

\section{2- Histopathological results}

\section{2-1- Gross pathological examination}

In control groups (group I and group II) examination revealed normal gross appearance of the liver and brain. In scald injured group (group III) liver and brain showed near normal gross appearance at 6 and 12 hours post scald injury. While at 24 hours examination, liver was enlarged, dark red in color with focal dark red areas seen on its surface. The brain revealed moderate edema of the meninges and areas of congestion.

\section{2-2-Microscopic examination}

\section{a- Liver (Table-2)}

Liver tissue of control groups (group I and II) showed that the liver architecture was preserved. The hepatic lobule was hexagonal in shape, consisting of radically arranged cords of hepatocytes radiating from central vein. These cords were separated by blood sinusoids (Figure-1a). While liver sections of scald injured group (group III) revealed significant pathological changes as aggregations of inflammatory cells in between the liver cords (Figure-1b), congestion and dilatation of blood vessels, focal inflammation in portal area with septal inflammation, (Figure-1c), vaculated hepatocytes with darkly stained nuclei and hydropic degeneration (Figure1d).

\section{b- Brain (Table-3)}

Examination of sections from the brain tissue of control groups (group I and II) showed normal neuronal structure formed of nerve cells with pale nuclei (Figure-3a). While the sections of scald injured group (group III) revealed significant pathological changes. The sections from rats 6 and $12 \mathrm{~h}$ after scald injury showed shrunken, vacuolated nerve cells with darkly stained nuclei and neuropil edema (Figure-3b,c). The sections from rats $24 \mathrm{~h}$ after scald injury showed progress in histopathological changes as increased number of shrunken neurons with darkly stain nuclei and neuropiledema (Figure$3 d)$.

\section{3- Immunohistochemical results}

The immunohistochemical examination of liver and brain tissue of control groups showed negative immunoreactions for (TNF- $\alpha$ ) (Figure-2a and 4a). While there were time dependent changes in (TNF- $\alpha$ ) activation in the liver and brain of group III. Positive staining was detected in the brain and liver of group III at 6 hours post injury and there were progress in the strength of the immunoreactions till 24 hours post injury (Figure-2b, c, d and 4b, c, d). 
Table-1: ANOVA and LSD statistical analysis of serum AST, ALT and ALKP levels in (U/L) at 6, 12 and 24 hours post injury within Group I (negative control), Group II (sham group) and group III (scald injured)

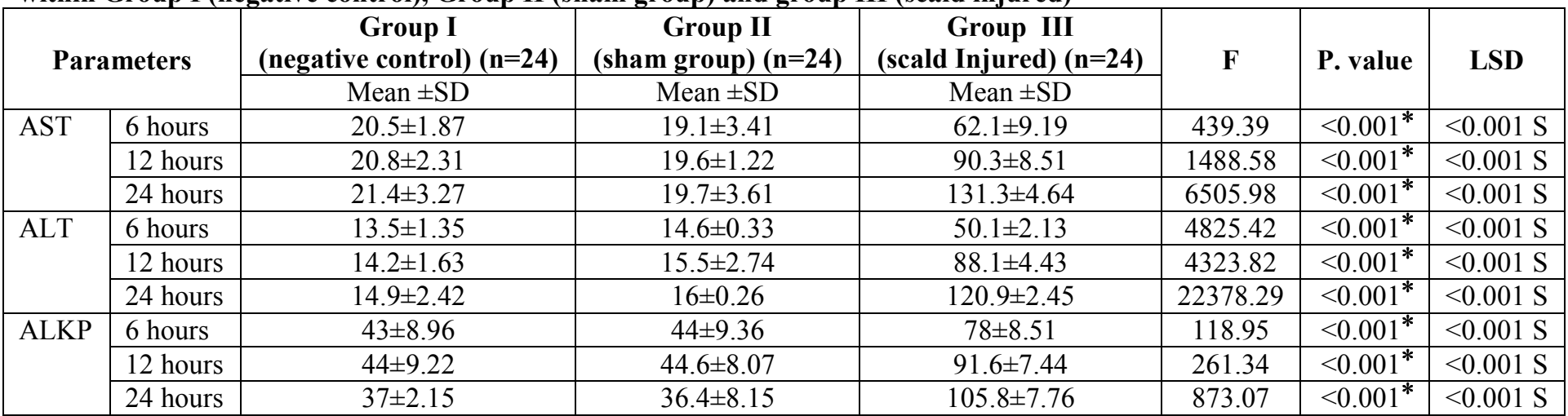

Data are expressed in terms of mean and standard deviation $(X+S D)$. Significant increase compared to control groups at $p<0.001$

Table-2: Histopathological examination of rat liver of the control group and group III at the different time points of the study $(6,12$ and 24 hours) using chi-square test

\begin{tabular}{|c|c|c|c|c|c|c|c|c|c|c|c|}
\hline \multirow{3}{*}{\multicolumn{2}{|c|}{ Histopathological changes }} & \multirow{2}{*}{\multicolumn{2}{|c|}{ Control groups }} & \multicolumn{6}{|c|}{ Group III (scald injury ) $(\mathrm{n}=24)$} & \multirow{3}{*}{$\mathbf{X} 2$} & \multirow{3}{*}{$P$ value } \\
\hline & & & & \multicolumn{2}{|c|}{$6 \mathrm{~h}(\mathrm{n}=8)$} & \multicolumn{2}{|c|}{$12 \mathrm{~h}(\mathrm{n}=8)$} & \multicolumn{2}{|c|}{$24 h(n=8)$} & & \\
\hline & & No. & $\%$ & No. & $\%$ & No. & $\%$ & No. & $\%$ & & \\
\hline \multirow[t]{2}{*}{ Cloudy swelling } & Focal & 0 & 0.0 & 0 & 0.0 & 3 & 37.5 & 5 & 62.5 & 12.0 & $0.007^{*}$ \\
\hline & Diffuse & 0 & 0.0 & 0 & 0.0 & 2 & 25.0 & 4 & 50.0 & 9.03 & $0.029^{*}$ \\
\hline \multirow[t]{2}{*}{ Hydropic Degeneration } & Focal & 0 & 0.0 & 0 & 0.0 & 1 & 12.5 & 3 & 37.5 & 6.86 & 0.077 \\
\hline & Diffuse & 0 & 0.0 & 0 & 0.0 & 1 & 12.5 & 5 & 62.5 & 13.95 & $0.003^{*}$ \\
\hline \multirow[t]{3}{*}{ Portal artery Inflammation } & Focal & 0 & 0.0 & 1 & 12.5 & 3 & 37.5 & 5 & 625 & 16.15 & $0.001^{*}$ \\
\hline & Septal inflammation & 0 & 0.0 & 2 & 25.0 & 5 & 62.5 & 7 & 87.5 & 14.73 & $0.002^{*}$ \\
\hline & Sever and Expansion & 0 & 0.0 & 0 & 0.0 & 2 & 25.0 & 6 & 75.0 & 16.0 & $0.001^{*}$ \\
\hline \multicolumn{2}{|l|}{ Kuppfer Cell Hyperplasia } & 0 & 0.0 & 0 & 0.0 & 3 & 37.5 & 4 & 50.0 & 9.33 & $0.025^{*}$ \\
\hline
\end{tabular}

$P$ value of $>0.05=$ Non-Significant. $P$ value of $<0.05=$ Significant $(*)$.

Table-3: Histopathological examination of rat brain of the control groups and group III at the different time points of the study $(6,12$ and 24 hours) by using chi-square test.

\begin{tabular}{|c|c|c|c|c|c|c|c|c|c|c|}
\hline \multirow{3}{*}{ Histopathological changes } & \multirow{2}{*}{\multicolumn{2}{|c|}{ Control group }} & \multicolumn{6}{|c|}{ Group III (scald injury ) $(n=24)$} & \multirow{3}{*}{$\mathbf{X} 2$} & \multirow{3}{*}{$P$ value } \\
\hline & & & \multicolumn{2}{|c|}{$6 h(n=8)$} & \multicolumn{2}{|c|}{$12 \mathrm{~h}(\mathrm{n}=8)$} & \multicolumn{2}{|c|}{$24 h(n=8)$} & & \\
\hline & No. & $\%$ & No. & $\%$ & No. & $\%$ & No. & $\%$ & & \\
\hline Pyknotic nuclei & 0 & 0.0 & 1 & 12.5 & 3 & 37.5 & 6 & 75.0 & 12.22 & $0.007^{*}$ \\
\hline Shrunken neurons & 0 & 0.0 & 2 & 25.0 & 4 & 50.0 & 7 & 87.5 & 13.86 & $0.003^{*}$ \\
\hline Neuropil edema & 0 & 0.0 & 2 & 25.0 & 3 & 37.5 & 6 & 75.0 & 10.39 & $0.016^{*}$ \\
\hline
\end{tabular}

$P$ value of $>0.05=$ Non-Significant. $P$ value of $<0.05=$ Significant $(*)$. 


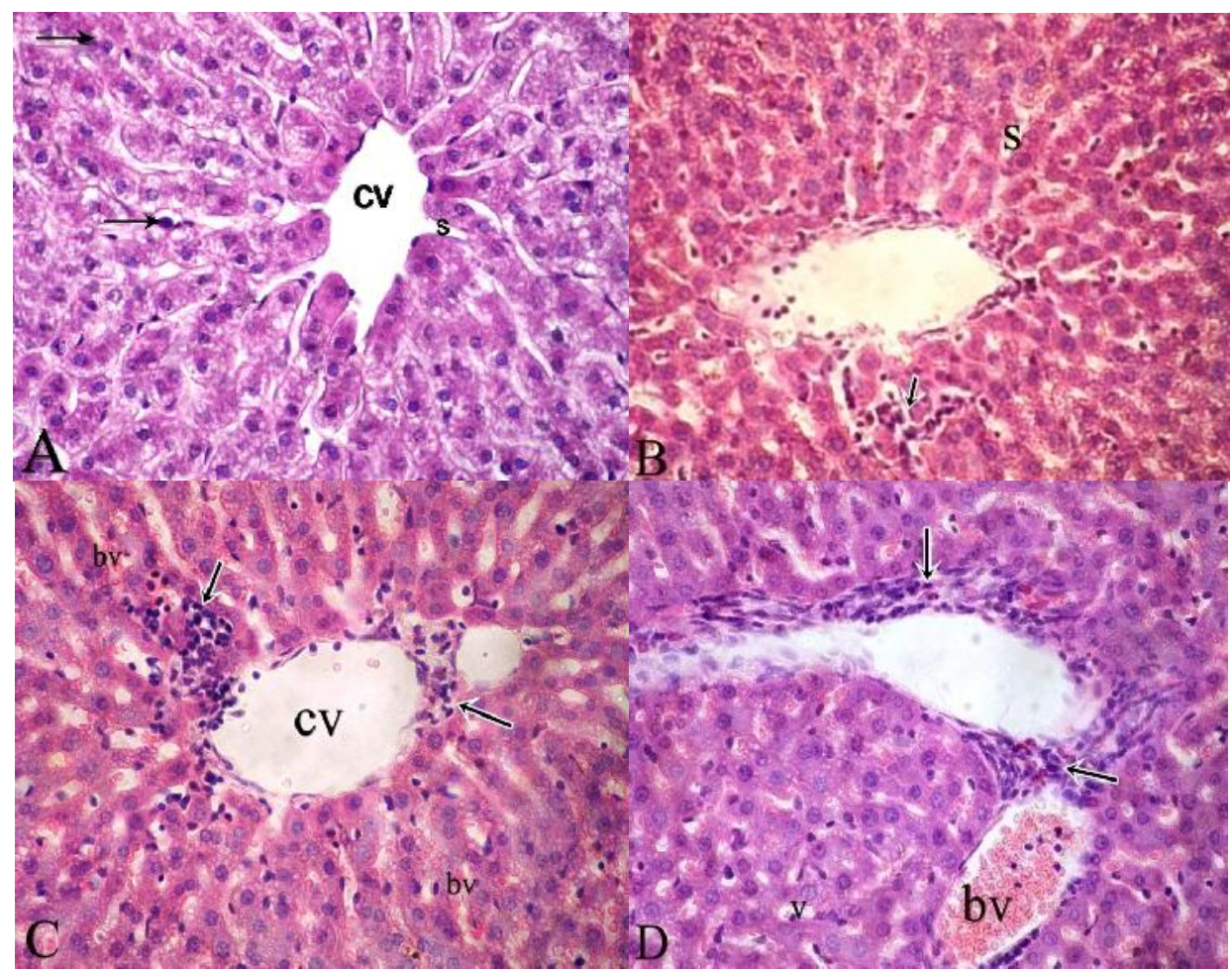

Figure-1: A photomicrograph of a section in the liver obtained from an adult male albino rat (A) control group showing plates of hepatocytes (arrows) radiating from central vein (cv) and separated by blood sinusoids (s), (B) group III 6h after scald showing of hepatocytes separated by dilated blood sinusoids (s) and inflammatory cell infiltration among cells (arrow), (C) section of group III 12h after scald showing dilated central vein (cv), peri central inflammatory cell infiltration (arrow) and dilated, congested blood vessels (bv), (D) group III $24 \mathrm{~h}$ after scald showing marked inflammatory response in portal area ( arrow) with marked blood vessels congestion (bv). Hepatocytes appear vaculated (v) (H\&E X400).

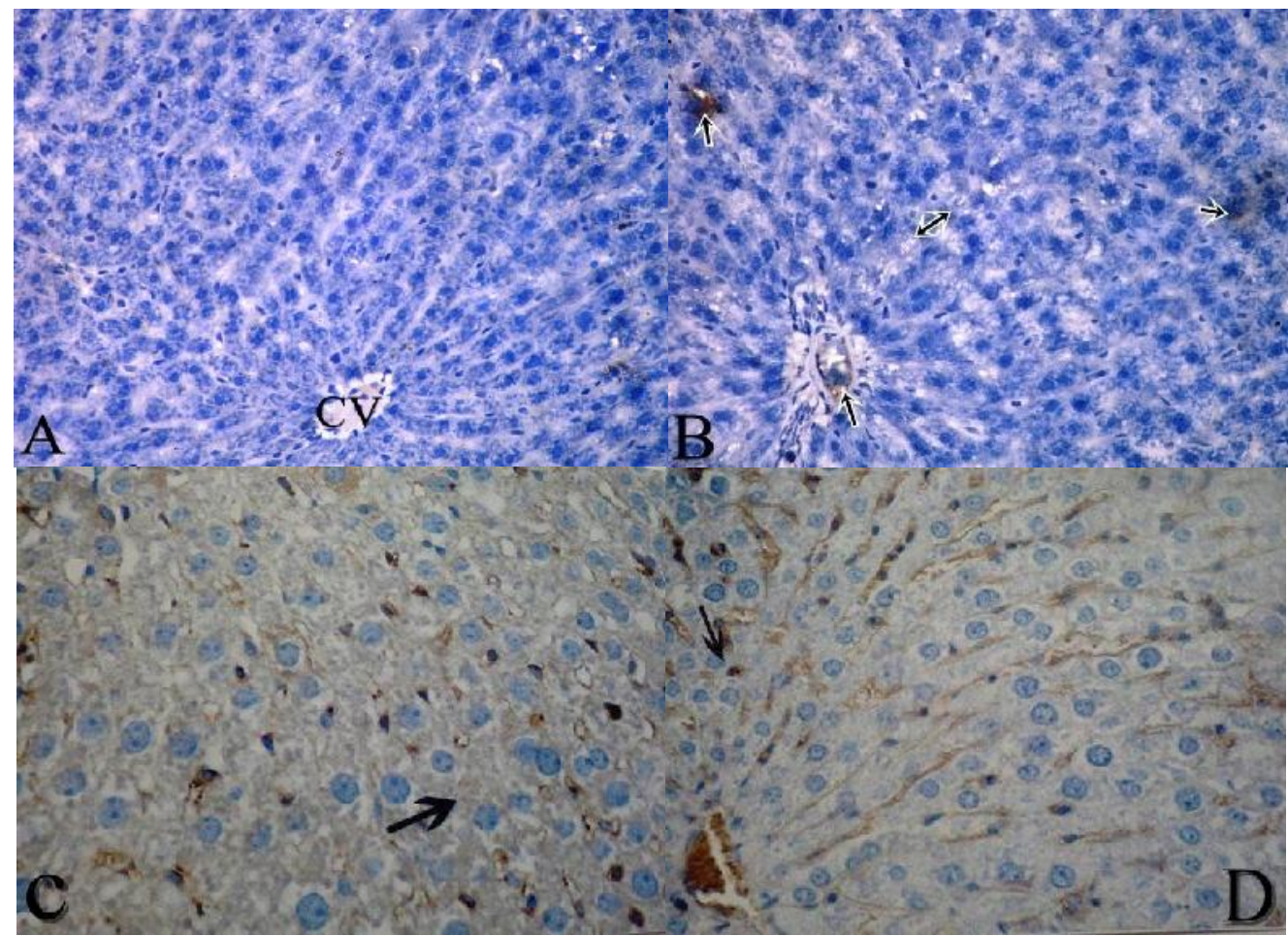

Figure-2: A photomicrograph of a section in the liver obtained from adult male albino rats immunohistologically stained for TNF- $\alpha$. (A) control group showing negative immunoreaction for TNF- $\alpha$, (B) group III $6 \mathrm{~h}$ after scald showing negative reaction for TNF- $\alpha$ in some cells (double head arrow) and positive in others (arrow), (C) group III 12h after scald showing increase number of Positive immunoreaction for TNF- $\alpha$, (D) group III $24 \mathrm{~h}$ after scald showing increase the reaction intensity for TNF$\alpha(\mathbf{X 4 0 0 )}$. 


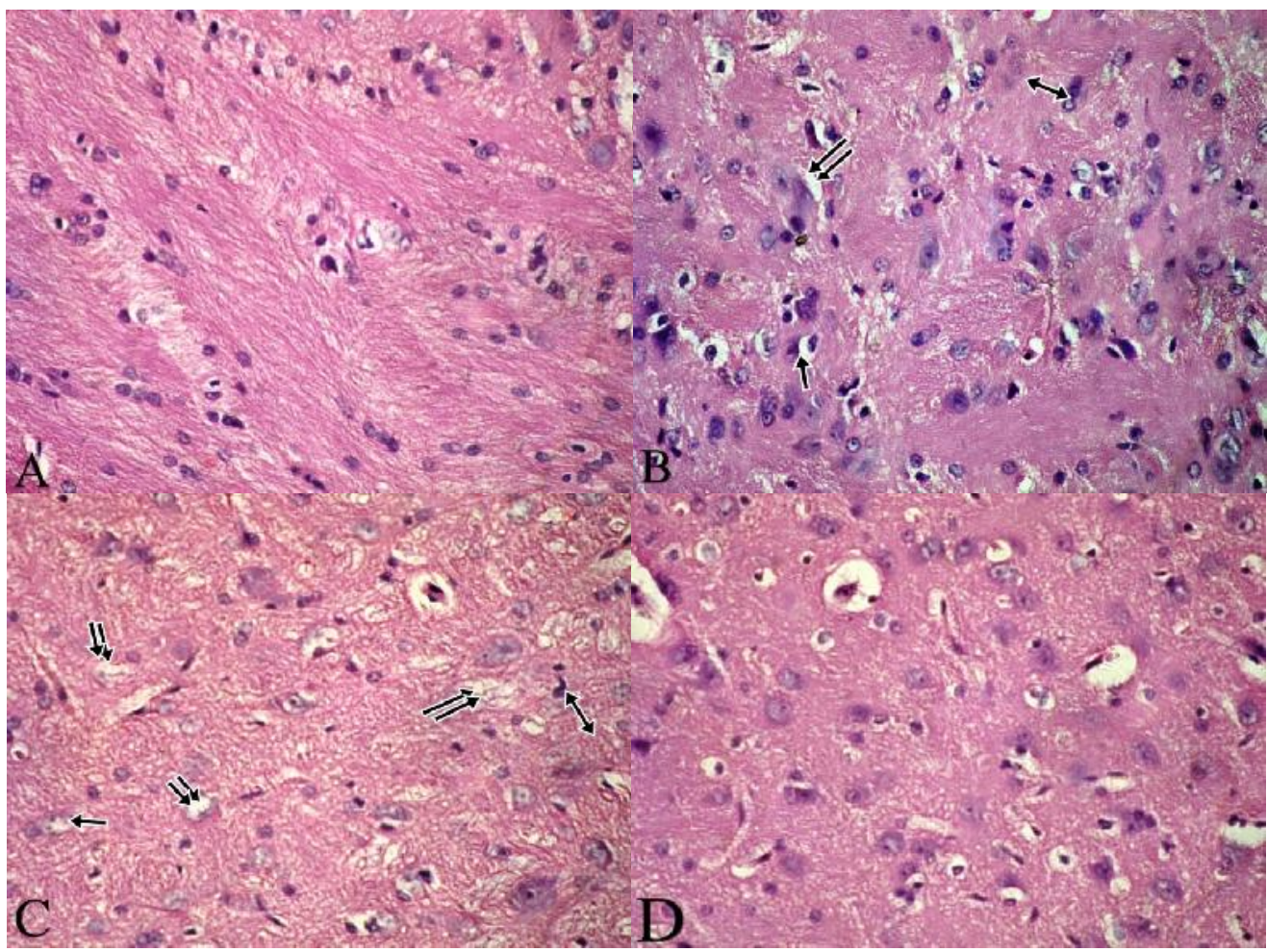

Figure-3:A photomicrograph of a section in the brain obtained from an adult male albino rat.(A) control group showing normal neuronal structure, (B) Section of group III 6 h after scald showing vaculated shrunken neurons (arrow) with darkly stained pyknotic nuclei(double head arrow) and neuropiledema (double arrow), (C) group III 12 h after scald showing shrunken neurons (arrow) with pyknotic nuclei (double head arrow) and neuropiledema (double arrows), (D) group III 24 h after scald showing increased number of shrunken neurons with darkly stain nuclei (H\&E stain X400).

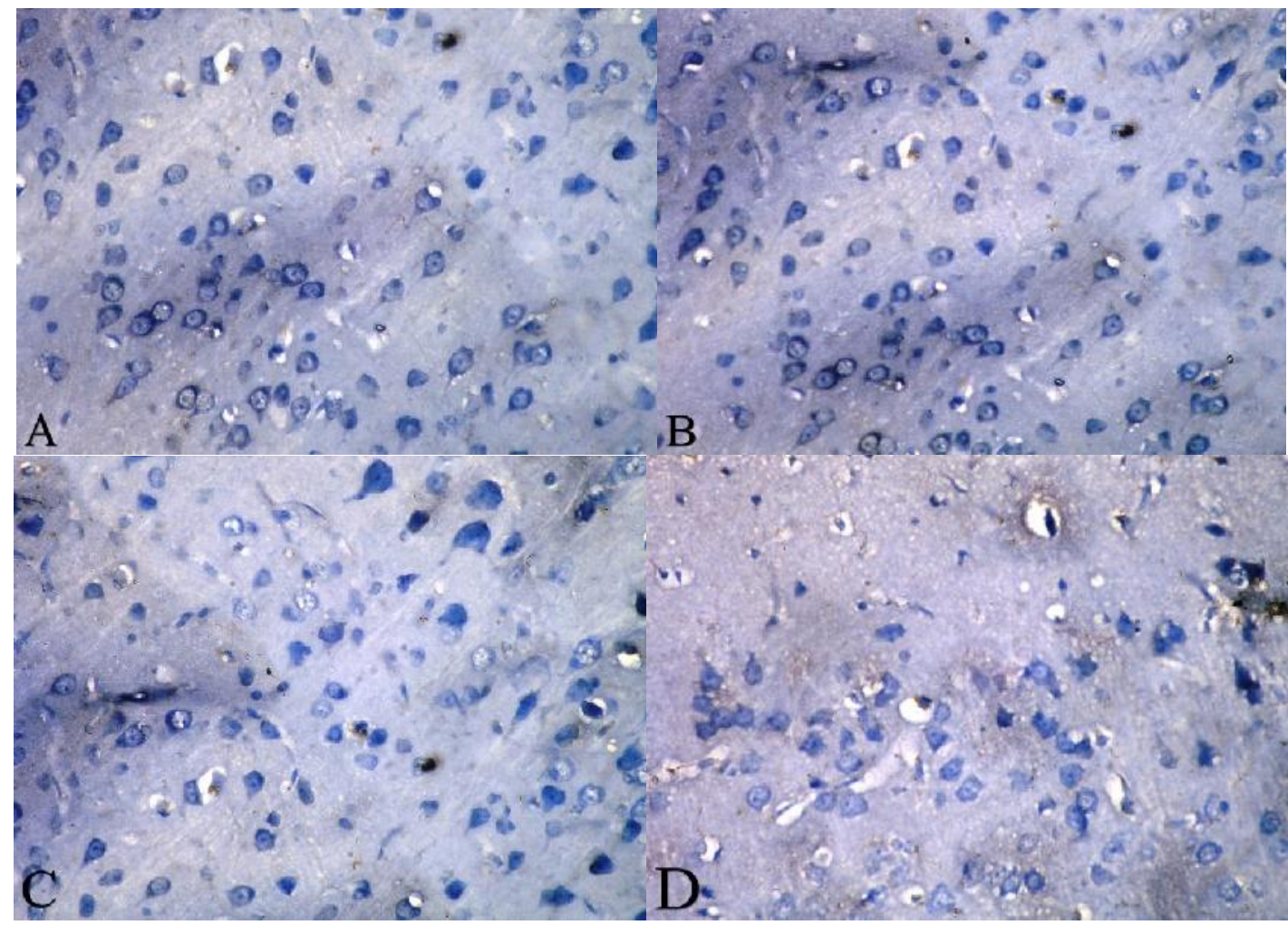

Figure- 4: A photomicrograph of a section in the brain obtained from adult male albino rats immunohistologically stained for TNF-a. (A) Control group showing negative. Immunoreaction for TNF-a, (B) Group III 6h after scald showing negative reaction for TNF- $\alpha$ in some cell's and positive in others, (C) Group III 12 h after scald showing increase number of Positive immunoreaction cells for TNF- $\alpha$, (D) Group III $24 \mathrm{~h}$ after scald showing strong positive immunoreaction for TNF- $\alpha$ (X400). 


\section{Discussion}

Thermal injury is a momentous cause of mortality and disability around the world. It is one of the most severe forms of trauma triggers a number of physiologic responses, including local and systemic inflammation, hypermetabolism, immune-suppression, and eventually organ dysfunction. This failure of different organ systems is thought to be due to increased apoptotic cell death and enhanced production of inflammatory cytokines such as interleukin-6 (IL-6), tumor necrosis factor-alpha (TNF- $\alpha$ ), interleukin 1beta (IL-1 $\beta)$, and prostaglandins (Grunwald and Garner, 2008).

Clinical studies have shown that an uncontrolled and prolonged action of inflammatory cytokines, which is evidenced by a sustained release of acute phase proteins, may contribute to detrimental complications after thermal injury (Yang et al., 2012).

The results of the present study showed a marked affection and disturbance in liver functions of group III (scald injured group) represented by significant increases in serum AST, ALT and ALKP levels as compared to control groups (group I and group II). Also, Serum levels of AST, ALT and ALKP increased significantly after 6 hours of scald injury till 24 hours.

These results are in accordance with Jeschke et al., (2001) who observed that serum AST, ALT, and ALKP significantly increased after scald when compared with controls. Also, serum AST and ALT peaked during the first day post-scald and ALKP during the second day post-scald; however, all enzymes returned to baseline between 5 and 7 days post-scald.

Furthermore, Jeschke (2009) have reported that thermal injury causes liver damage by edema formation, hypoperfusion, pro-inflammatory cytokines, or other cell death signals with the release of the hepatic enzymes. Serum AST, ALT and ALKP are elevated between $50 \%$ to $200 \%$ from baseline when compared with normal levels.

The results of the present study revealed that thermal injury induced pathological changes in the liver as aggregations of inflammatory cells in between the liver cords, congestion and dilatation of blood vessels, focal inflammation in portal area with septal inflammation, vaculated hepatocytes with darkly staied nuclei and hydropic degeneration.

The same finding were obtained by İseri et al., (2008) who observed sever damage in the liver of rats in the form of vacuolization of hepatocytes, dilation and vascular congestion in sinusoids and increased the number of Kupffer cells after the scald insult.

Recent studies suggest that thermal injury can change liver morphology in rats and decreases concentrations of protein and DNA. Previous studies indicated that a cutaneous injury can signal changes in gene expression by the liver suggesting that this organ plays a pivotal role in immune function, inflammatory processes, and the acute phase response to scald. Also, the synthesis of constitutive hepatic proteins, acute phase proteins, cyokines, and other mediators by the livers make it a determining factor in scald survival (Zheng et al., 2006).

The immunohistochemical examination of liver tissue in this study showed time dependent changes in (TNF- $\alpha$ ) activation in the liver tissue of scald injured group. Positive staining was detected in liver at 6 hours post injury and there were progress in the strength of the immunoreactions till 24 hours.

In Parallel with these results, Wang et al. (2006) observed an increase in the expression of TNF- $\alpha$ mRNA which arrived to its peak at 12 hours post scald injury and lasted till 48 hours.

Jeschke et al. (2008) have concluded that after a thermal injury, serum and hepatic concentration of proinflammatory cytokines such as IL- $1 \alpha / \beta$, IL- 6 , and TNF- $\alpha$ are increased and therefore, suggested two possible mechanisms could be involved in increased liver damage: decreased splanchnic blood flow; and elevation of pro-inflammatory cytokines.

Tumor necrosis factor alpha is one of the mononuclear factors created earliest by macrophages after scald or trauma, which is involved in immune defense against infection. Appropriate quantity of TNF- $\alpha$ has protective effects, but the marked increased TNF- $\alpha$ production in macrophages after scald is harmful, which can cause organ injury and negative nitrogen balance (Schwacha et al., 2001).

The results of the present study showed that brain sections of scald injured group (group III) revealed many pathological changes in the form of shrunken, vacuolated nerve cells with darkly stained nuclei and neuropiledema.

In consistent with these results Taran et al.(2005)have detected identifiable edema, compression of the meningeal teguments, moderate dissociation of the fibrous structures, and signs of stasis in average and small vessels, while performing histolopathgical examination of the cerebral substance of cases died shortly after scald injury .

Furthermore, in animal studies of scald injury, magnetic resonance imaging has identified marked changes in the brain up to 3 days post-scald injury, most notably brain swelling and lesions. Over the initial 24 hour post-scald period, rats have been found to display decreased glucose utilization in the brain indicating an acute dysregulation of glucose metabolism in the brain (Li et al., 2001).

Scald-induced neuroinflammation continues to be an underestimated entity in the critically ill scald patient. If the blood-brain barrier (BBB) is breached, systemic inflammatory molecules and phagocytes readily enter the brain and activate sessile cells of the central nervous system. Copious amounts of reactive oxygen species, reactive nitrogen species, proteases, cytokines/chemokines, and complement proteins are being released by these inflammatory cells, resulting in neuronal damage and life-threatening cerebral edema (Flierl et al., 2009).

Neuropsychological disorders have been documented in children following scald injury. In these children, chronic mental disability was correlated with the size of the scald injury. Additionally, a retrospective clinical study found that a small percentage of scald patients also suffer from acute stroke (Cho et al., 2007).

The results of the present study revealed time dependent changes in (TNF- $\alpha$ ) activation in brain tissue of group III. Positive staining was detected in the brain at 6 hours 
after scald injury and there were progress in the strength of the immunoreactions till 24 hours post injury.

The results of the present study coincided with Gatson et al. (2009) who found out that severe scald injury induced an extremely rapid and substantial increase in the levels of TNF- $\alpha$, IL-1 $\beta$, and IL- 6 in the rat brain tissue.

Also, the data presented in the study of Guyon et al. (2008) showed that profound increase in brain proinflammatory cytokines was due to local brain synthesis in glia and neurons or from peripherally produced cytokines that penetrated into the brain from the systemic circulation following severe scald injury.

Cytokines and chemokines are initially only produced at the scald site, but may become systemic and directly attack the BBB. In addition, severe scald injury significantly up-regulates mRNA levels of TNF- $\alpha$, IL- $1 \beta$ and intercellular adhesion molecule-1 in brain tissue as early as 3 hours post injury (Reyes et al., 2006).

\section{Conclusion}

From the previous results it can be concluded that, scald injury induced a noticeable affection in liver functions, which was characterized by significant increases in serum ALT, AST and ALKP. Histopathological changes in liver and brain. Also, a time dependent (TNF- $\alpha$ ) activation was detected in liver and brain of adult male albino rats. Further studies should be directed to detect the reversibility of such changes to be taken into account by burn units during treatment of the victims of such thermal injury. A comparative study between such changes with scald and dry scald injury is also recommended.

More detailed studies should be directed towards detection of other possible effects of scald injuries on remote organs at different time interval after injury in order to predict possible causes of mortality and disabilities of scald injury victims.

\section{References}

Adeteye OV, Yama OE, and Gbotolorun SC (2011): Third degree scalds in female Wister rats: the corollary on estrous cycle and ovarian histo-architectural organization. Inter J Med Medic Sc. 3:256-61.

Agay D, Andriollo-Sanchez M, Claeyssen R et al., (2008): Interleukin-6, TNF-alpha and interleukin-1 beta levels in blood and tissue in severely scalded rats. European cytokine network. 19(1): 1-7.

Bancroft J D and Gamble M (2002): Theory and practice of histological techniques.5th ed. Churchill Livingstone: New York, London, Pheladelphia.23:301-312.

Chen KH, Gu W, Zeng L, et al. (2011): Identification of haplotype tag SNPs within the entire TLR2 gene and their clinical relevance in patients with major trauma. Shock .35:35-41.

Cho SJ, Minn YK, and Kwon KH (2007): Stroke after scald. Cerebrovascular Diseases. 24(2-3): 261-263.

Farahat F (1999): Medicolegal aspect of hepatorenal brunt in scalds. pp: 1-2, 69-70.

Flierl MA, Stahel PF, Touban B et al., (2009): Bench-tobedside review: scald-induced cerebral inflammation-a neglected entity. Critical care. 13(3): 215.
Gatson JW, Maass DL, Simpkins JW et al., (2009): Estrogen treatment following severe scald injury reduces brain inflammation and apoptotic signaling. J Neuroinflammation. 6: 30 .

Grunwald TB and Garner WL (2008): Acute scalds. Plastic Reconstructive Surgery . 121:311e-319e.

Guyon A, Massa F, Rovere C et al., (2008): How cytokines can influence the brain: a role for chemokines? J Neuroimmunol. 198:46-55.

Happerfield LC, Bobrow LG, Bains RM et al., (1993): Peroxidase labeling immunocytochemistry: a comparison of eleven commercially available avidinbiotin systems. J HistochemCytochem. 41: 291-302.

İseri SÖ, Ersoy Y, Gedik N et al., (2008): Protective role of adrenomedullin in scald-induced remote organ damage in the rat. Regulatory peptides. 146(1): 99105.

Jeschke MG, Chinkes DL, Finnerty CC et al., (2008): The pathophysiologic response to severe scald injury. Annals of surgery. 248(3): 387.

Jeschke MG (2009): The hepatic response to thermal injury: is the liver important for post-scald outcomes?. Molecular Medicine. 15(9-10): 337.

Jeschke MG, Low JF, Spies M et al., (2001): Cell proliferation, apoptosis, NF- $\mathrm{B}$ expression, enzyme, protein, and weight changes in livers of scalded rats. American Journal of Physiology-Gastrointestinal and Liver Physiology. 280(6): G1314-G1320.

Jewo PI, Duru FI, Osinubi AA et al., (2011): Histological changes and testicular dysfunction in severely scalded rats. Macedonian Journal of Medical Sciences. 4(3): 227-233.

Li H, Ying D, Sun J et al., (2001): Comparative observation with MRI and pathology of brain edema at the early stage of severe scald. Chin J Traumatol. 4:226-230.

Nemzek J, Boloqos G, Williams B et al., (2001): Differences in normal values for murine white blood cells counts and other hematological parameters based on sampling site. Inflammation Research.50: 523-527.

Price LA, Thombs B, Chen CL et al., (2007): Liver disease in scald injury: evidence from a national sample of adult patients. J Scalds Wounds. 7: 31-338.

Reyes RJ, Wu Y, Lai Q et al., (2006): Early inflammatory response in rat brain after peripheral thermal injury. Neurosci Lett. 407:11-15.

Schwacha MG, Schneider CP, Bland KI et al., (2001): Resistance of macrophages to the suppressive effect of interleukin-10 following thermal injury. Am J Physiol Cell Physiology. 281(4):C1180-7.

Shah M, Orton E, Tata LJ et al., (2013): Risk factors for scald injury in children under 5 years of age: A casecontrol study using routinely collected data. Scalds. 39(7): 1474-1478.

Swann K, Berger J, Sprague SM et al., (2007): Peripheral thermal injury causes blood-brain barrier dysfunction and matrix metalloproteinase (MMP) expression in rat. Brain research. 1129: 26-33.

Taran A , Baciu N, Rafulea V et al., (2005): Clinical and autopsy diagnoses of visceral affections of patients who died because of complicated scalds with multi- 
organ failure. Annals of scalds and fire disasters. 18(4): 177.

Wang X., Wang Y, Peng D et al., (2011): Changes in the inositol lipid signal system and effects on the secretion of TNF- $\alpha$ by macrophages in severely scalded mice. Scalds. 37(8): 1378-1385.

Wang Y, Peng D, Huang W et al., (2006): Mechanism of altered TNF- $\alpha$ expression by macrophage and the modulatory effect of Panaxnotoginsengsaponins in scald mice. Scalds. 32(7): 846-852.
Yang, Q, Orman, MA, Berthiaume F et al., (2012): Dynamics of short-term gene expression profiling in liver following thermal injury. Journal of Surgical Research. 176(2): 549-558.

Zhang JP, Ying X, Liang WY et al., (2008): Apoptosis in cardiac myocytes during the early stage after severe scald. J Trauma. 65:401-408. discussion 408.

Zheng H, Chen XL, Han $Z$ et al., (2006): Effect of Ligustrazine on liver injury after scald trauma. Scalds. 32(3): 328-334.

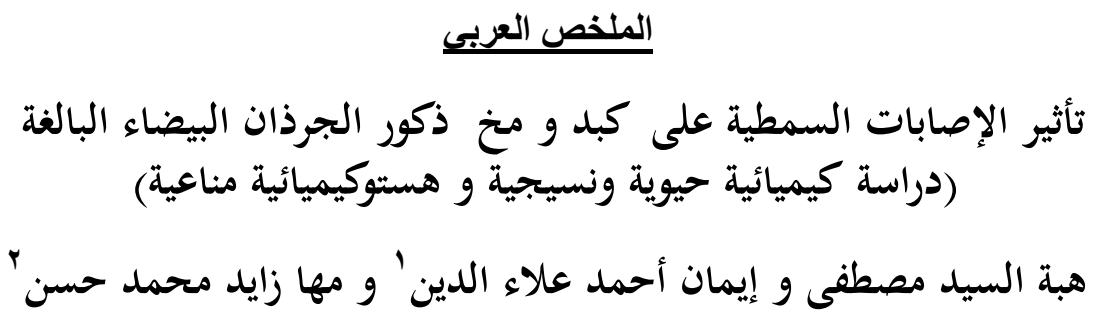

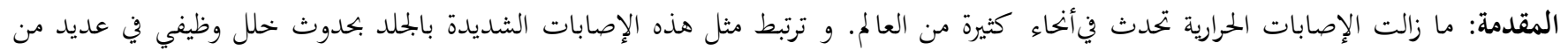

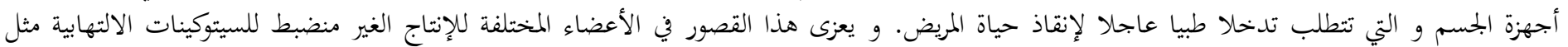

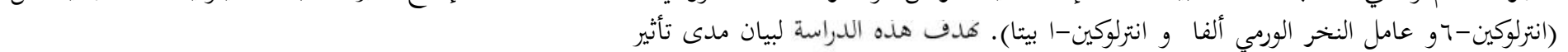

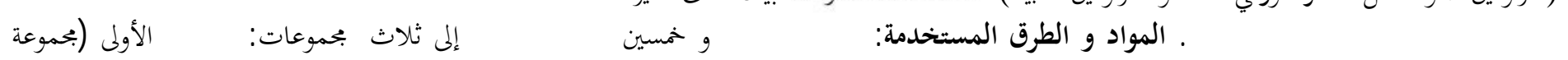

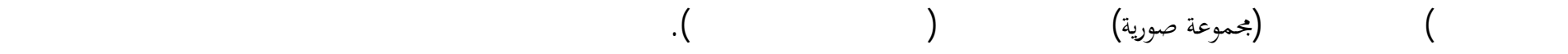

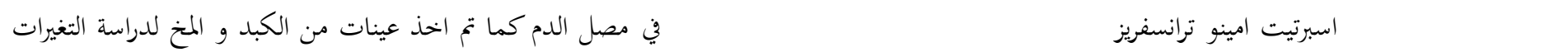

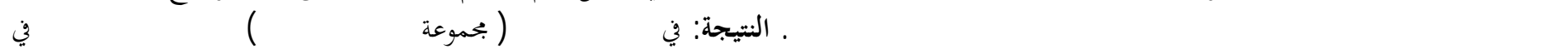

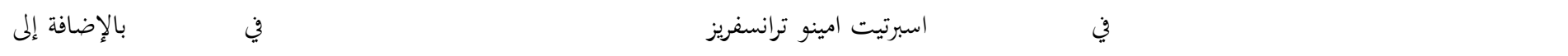

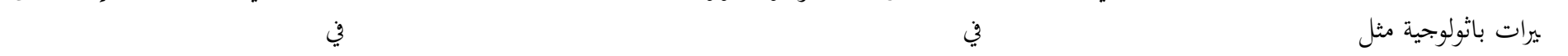

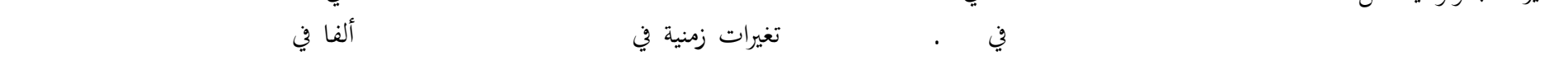

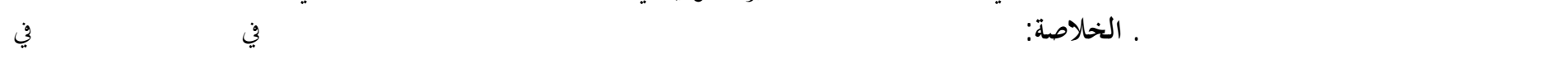

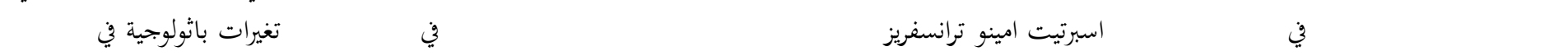

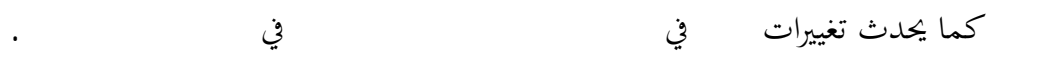

ا قسم الطب الثرعي والسموم الإكلينيكية - كلية الطب البشري - جامعة الزقازيق r قسم الهسنولوجيا و بيولوجيا الخلايا - كلية الطب البشري - جامعة الزقازيق 